\title{
Primary Hyperparathyroidism with Multiple Fractures and Brown Tumors in a 14-year-old Girl: Case Report \& Review of Literature
}

\author{
Nasim Khan, Nazmun Nahar, Rawshan Ara, Fahima Akter Dowel, Shakila Zaman Rima, Ratan Kumar Chakraborty, Nadiruzzaman, \\ Saiyeeda Mahmood, Gazi Abul Hossain \\ Institute of Nuclear Medicine and Allied Sciences (INMAS), Mymensingh Medical College Hospital Campus, Mymensingh-2200, Bangladesh
}

Correspondence Address: Dr. Nasim Khan, Professor \& CMO, INMAS, Mymensingh. nasim.inmas@gmail.com

\begin{abstract}
Primary hyperparathyroidism (PHPT) presenting as pathological fractures with brown tumors is very rare in children. The rarity and variable presentation of PHPT can make its diagnosis a challenge especially in the developing countries where malnutrition may mask hypercalcemia. We report a case of a 14-year-old girl who presented with muscle pain, multiple fractures and bone pain. Laboratory tests revealed normocalcemia with vitamin D insufficiency. Subsequent biochemical, radiographic and scintigraphic findings were consistent with primary hyperparathyroidism due to a parathyroid adenoma. Tumor resection was done in a local hospital and histopathology confirmed parathyroid adenoma. Following parathyroidectomy, fractures were treated conservatively with supplementation of calcium and vitamin D. We found her quite capable of self-care and walking in good health at 1 year follow up. Though rare, PHPT-causing brown tumors related skeletal fractures is an important differential diagnosis in the evaluation of patients presenting with multiple foci of radiotracer uptake in bone scintigraphy.
\end{abstract}

Keywords: Hyperparathyroidism, pathological fracture, brown tumor, parathyroid adenoma, bone scintigraphy

Bangladesh J. Nucl. Med. Vol. 22 No. 2 July 2019

DOI: https://doi.org/10.3329/bjnm.v22i2.51765

\section{INTRODUCTION}

Primary hyperparathyroidism (PHPT) is rare in childhood and early adolescence, and may present special difficulties in diagnosis (1). The clinical symptoms of hyperparathyroidism at an early stage are mostly nonspecific and atypical, making it very difficult to diagnose. The diagnosis of most cases is delayed and patients may present with complications of long-term hypercalcemia, such as brown tumors, or nephrocalcinosis or pathological fracture. Although the measurement of serum calcium and parathyroid hormone (PTH) levels provide early diagnosis and decreases the incidence of radiographic bone involvement, progressive major bone lesions may still be seen in developing countries even in the modern era. The diagnosis can be very perplexing especially because the expected hypercalcemia associated with the PHPT may be masked by calcium, protein or vitamin D deficiency $(2,3)$. The reported incidence of fractures in hyperparathyroidism is quite low, about $10 \%$ in two large series (4), and apart from vertebral compression fractures, no characteristic fracture pattern have been described. Extensive bony involvement with pathological fractures as a presenting feature due to parathyroid carcinoma has been documented $(2,5)$, but multiple pathological fractures, as a presenting feature of primary hyperthyroidism due to parathyroid adenoma is extremely rare. Here we present a case of an adolescent girl who presented with multiple fractures and brown tumors due to parathyroid adenoma.

\section{CASE REPORT}

A 14-years-old girl was referred to the scintigraphy division of Institute of Nuclear Medicine \& Allied Sciences (INMAS), Mymensingh for the evaluation of recurrent fractures and bone tumors. She had been experiencing muscle weakness, bone pain and recurrent bone fractures of the limb over 2-years following minor accidental injury. There was history of fracture of her right mid femur which was not treated as pathological fracture. Inadequate and improper management led to healing with malunion and limb deformity. Prior to this, she had left femoral neck fracture managed by conservative treatment two years ago. She also recalled one-year history of both thigh pain 
unresponsive to common analgesics, unexplained fatigue and generalized muscle weakness. Physical examination revealed right thigh and right humerus swelling with tenderness, deformity of left hip with movement restrictions. The girl had no history of tuberculosis, rickets or childhood malnutrition. Radiographic images showed multiple fractures of left femur neck, shaft of right femur and ribs with features of osteolysis (Figure 1).

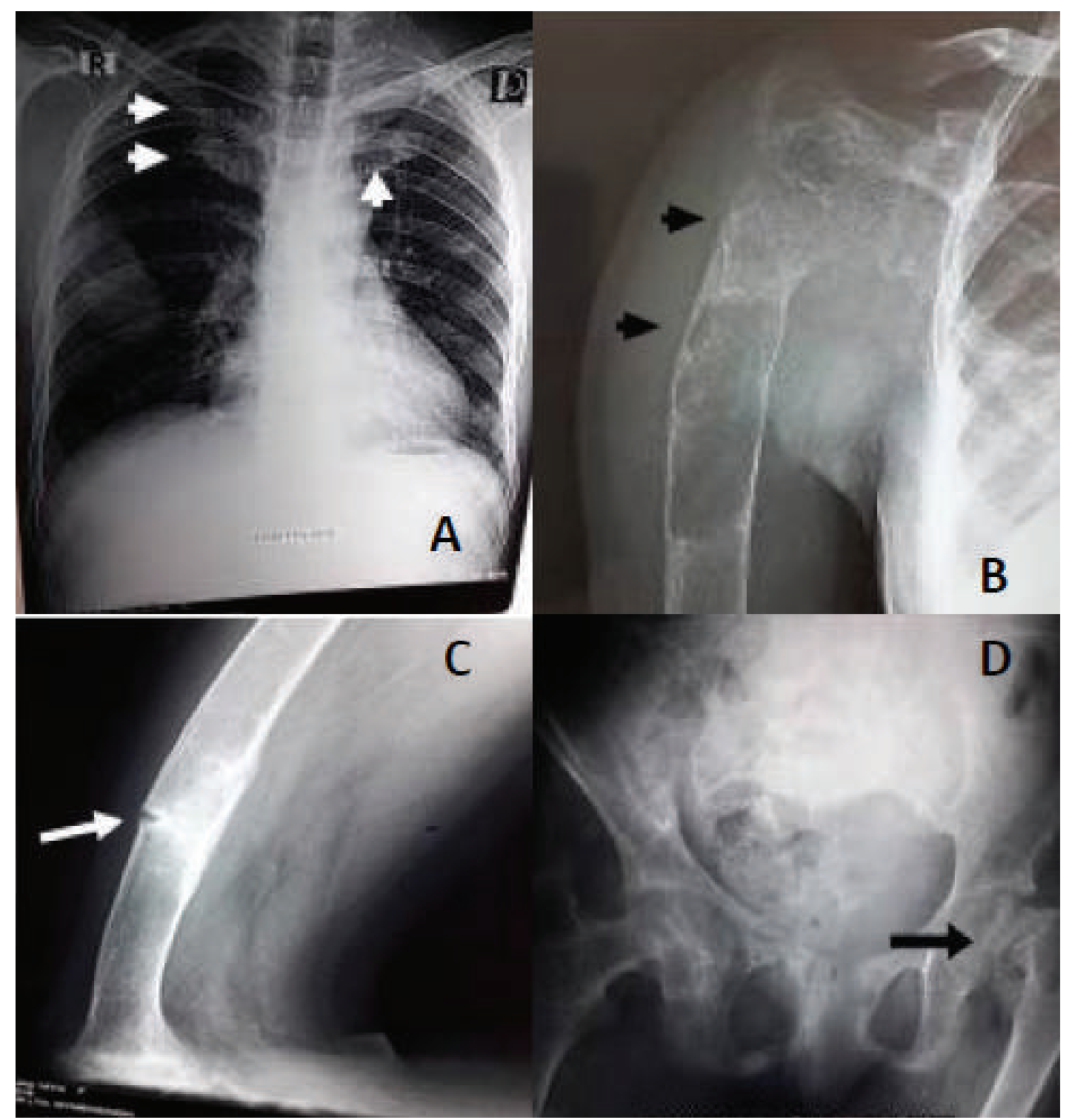

Figure 1: (A) Chest X-ray PA view showing multiple rib fracture and osteolytic lesions (white arrow heads). (B) Right humerus and shoulder with expansile osteolytic lesions and thinning of cortex (black arrow heads). (C) Right femur showing both sclerotic and osteolytic lesions with pathological fracture at the lower mid shaft (white arrow). (D) Pelvic $\mathrm{X}$-ray shows pathological fracture in the neck of left femur (black arrow) with pelvic deformity.

Initial laboratory tests showed normal complete blood count, serum calcium level of $10.2 \mathrm{mg} / \mathrm{dl}$, (normal range $10.0-12.0 \mathrm{mg} / \mathrm{dl}$ ), low phosphorus level of $2.4 \mathrm{mg} / \mathrm{dl}$, (normal range, 3.0-5.0 $\mathrm{mg} / \mathrm{dl}$ ), and slightly high alkaline phosphatase (ALP) 380 IU/L (normal range, 65-300 IU/L). HBsAg and RA tests were negative.
A whole body bone scan was performed with $555 \mathrm{MBq}$ of 99mTc-methylene diphosphonate (99mTc-MDP) using Symbia True Point SPECT/CT. The bone scan showed multiple focal areas of increased radiotracer uptake in the right humerus, right scapula, sternum, clavicles and ribs bilaterally, left femoral neck, right femoral shaft and lower part of left tibia (Figure 2). 


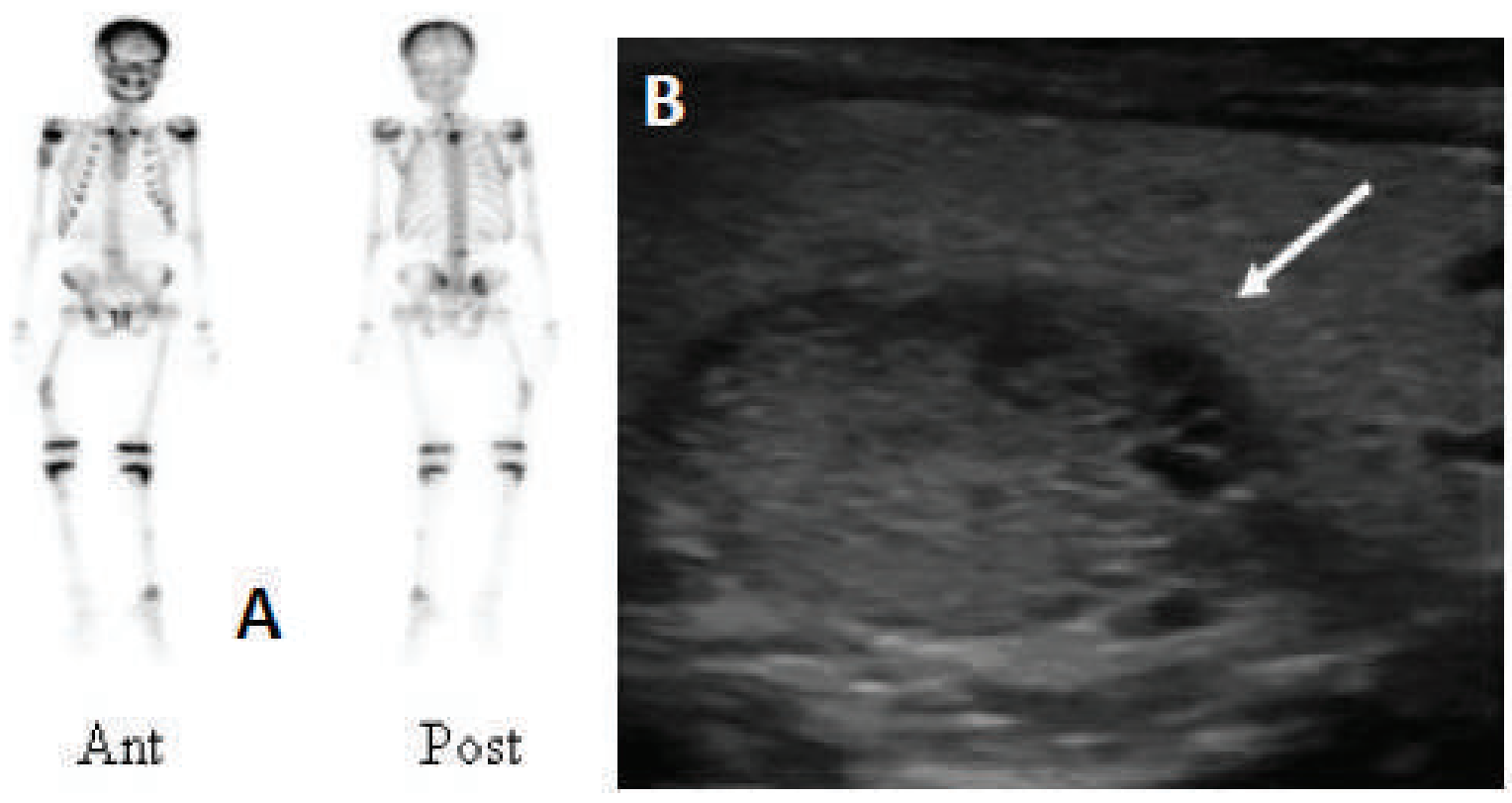

Figure 2: (A) Whole body bone scan with 99mTc - MDP showing mulitfocal lesions involving right humurs, bilateral clavicles, vertebrae, femur and tibial bones. (B) High resolusion neck ultrasonography shows a nodular mass ( 1.8 $X 0.7 \mathrm{~cm}$ ) in the lower part of left thyroid lobe. The mass was hypoechoic and almost homogenous in echotexture.

Scintigraphic images looked like extensive skeletal metastases of unknown primary (SMUP). However, cancer biomarkers (e.g. Serum CEA, CA-15-3 and CA-125), abdominal ultrasound scan, kidney and thyroid function tests were unremarkable. After getting the elevated serum parathormone (PTH) level of $356 \mathrm{ng} / \mathrm{dl}$ (normal range, $<20 \mathrm{ng} / \mathrm{dl}$ ) and low 25-hydroxyvitamin $\mathrm{D}$ (25-OHD) level of $21.0 \mathrm{ng} / \mathrm{ml}$ (normal range, $30-100$ $\mathrm{ng} / \mathrm{ml}$ ), a parathyroid imaging was scheduled for the

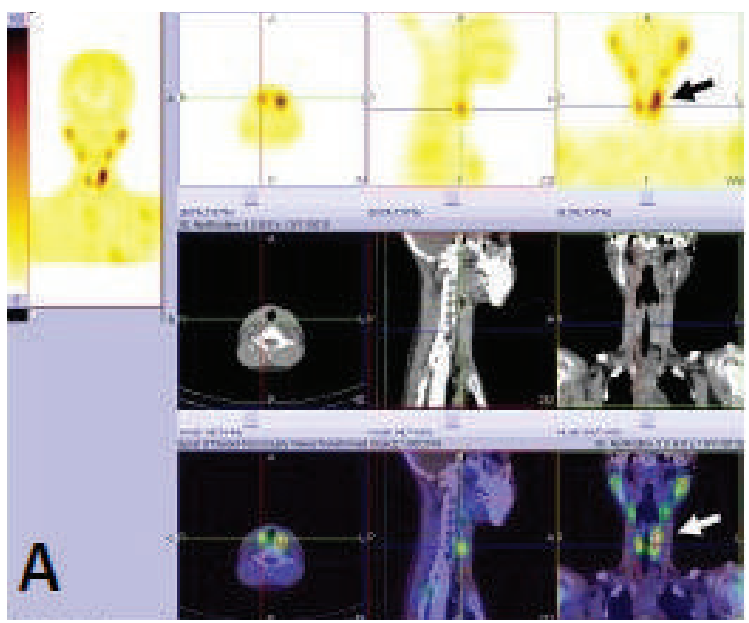

patient. Firstly, a high resolution ultrasound scan of neck revealed a small hypoechoic nodular mass like lesion $(\sim 1.8$ $\mathrm{X} 0.7 \mathrm{~cm}$ ) within the lower part of left lobe of thyroid with possible extension cephalad and posterior, and thought to be a thyroid nodule (Figure 2). However, the possibility of an intrathyroidal parathyroid tumor could not be excluded. Secondly, a 99mTc-MIBI- SPECT/CT scan of neck and upper chest showed MIBI avid left inferior parathyroid lesion highly suspicious of adenoma (Figure 3).

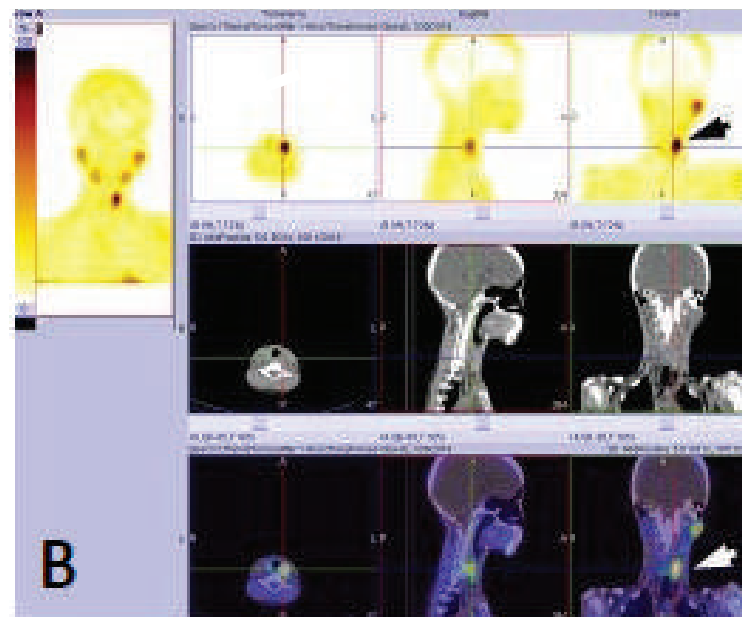

Figure 3: (A) Early image of dual phase 99mTc-MIBI SPECT/CT shows well developed thyroid gland, increased radiotracer uptake at the lower pole of the left thyroid lobe (black arrow). (B) Delayed image of the SPECT/CT scan shows decreased radioactivity in the thyroid gland, high radiotracer uptake is still revealed at the lower part of the left thyroid lobe (arrow head) coincided with a mass in parathyroid gland through CT. Hybrid SPECT/CT confirms left parathyroid adenoma. 

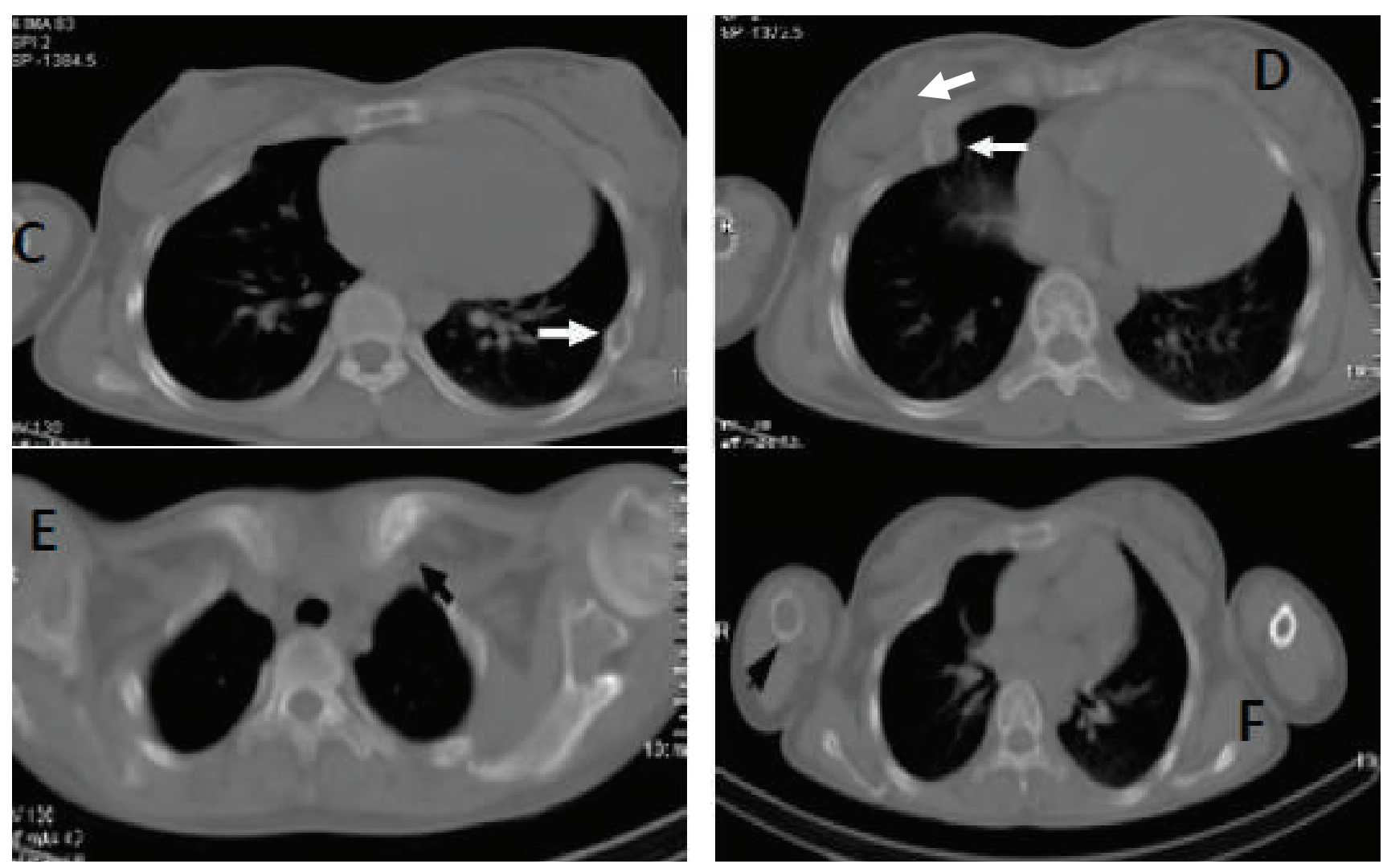

Figure 3: (C, D) Axial images of the CT component of SPECT/CT demonstrate expansile osteolytic lesion involving a rib (white arrow), and a healed rib fracture with deformity (thin white arrow). (E, F) Axial images of the CT of upper chest reveals osteolysis with erosion of the medial end of both clavicles (black arrows), and centrally located intramedullary osteolytic lesion with thinning of cortex at upper part of right humerus (black arrow head).

In addition, CT component of SPECT/CT demonstrated multiple osteolytic and expansile bone lesions in sternum, clavicles, multiple ribs and right humerus (Figure 3). Subsequently, patient underwent left inferior parathyroidectomy with curative intent. Histopatho- logical examination confirmed the diagnosis of parathyroid adenoma and no features of malignancy.

Two days postoperatively, the patient experienced transient mild hypocalcemia $(7.1 \mathrm{mg} / \mathrm{dl})$ and treated with oral calcium carbonate supplementation. Interestingly, on 7th postoperative day, there was persistently elevated PTH (273 $\mathrm{ng} / \mathrm{dl}$ ) which raised the suspicion of either incomplete surgical procedure or the presence of more adenoma. Whole body imaging with 99mTc-MIBI SPECT/CT was done which apparently showed no abnormality but CT component revealed osteolytic and expansile bony lesions in the sternum, clavicle, ribs, right humerus, and additionally, similar lesions in left femoral neck and right femur (Figure 4).
Decreased bone density was also observed and the trabecular bone of the femur was thinned. Histopathological biopsy of right femoral lesion reported features consistent with brown tumor and possibly the osteolytic expansile skeletal lesions of left femoral neck, right humerus, sternum and other sites were due to primary hyperparathyroidism-related multifocal brown tumors.

After conservative treatment of the fractures the patient was discharged with supplementation of oral calcium carbonate and cholecalciferol. However, PTH level was elevated, which took 3 months to come down to normal, indicating cure. Furthermore, patient neither complained bony pain nor muscle weakness.

At 1 year follow up, patient was found stable with CT proven regression of the skeletal lesions with a serum PTH of $15 \mathrm{ng} / \mathrm{ml}$ and continued calcium and vitamin-D supplementation. 

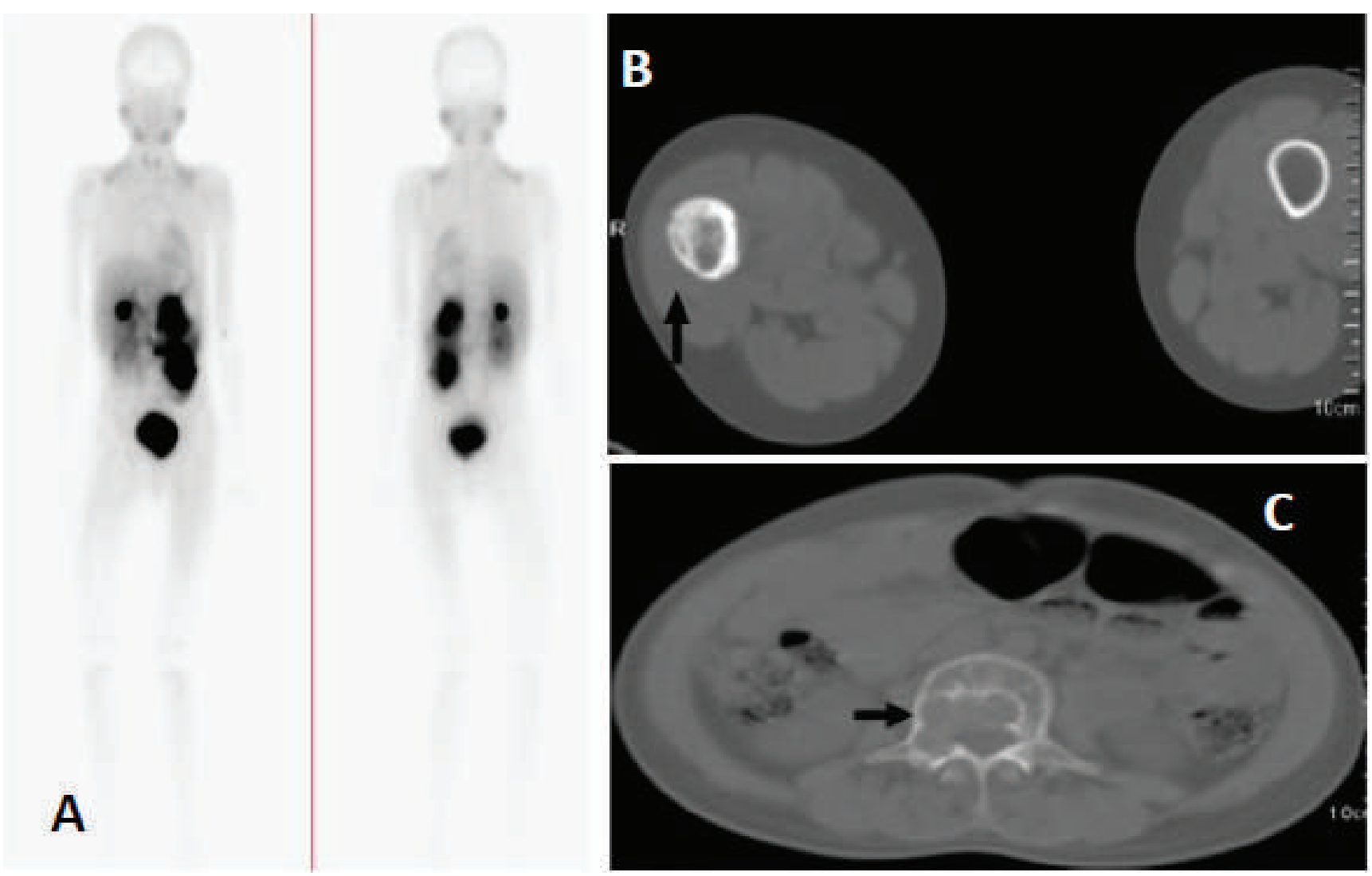

Figure 4: (A) Anterior \& posterior 99mTc-MIBI whole body images shows no abnormal radiotracer accumulation in the neck or any other region of the body. (B, C) Axial images of CT component of SPECT/CT show osteolytic lesions in the right femur and vertebrae (black arrows).

\section{DISCUSSION}

Primary hyperparathyroidism (PHPT) affects 22 per 100,0000 people per year with a mean age of onset of 56 years and a female preponderance of $3: 1$ (6). The incidence in patients aged 12 to 28 years is less than $5 \%$ (7). Solitary parathyroid adenomas account for 80 to $85 \%$ cases of PHPT. Less common causes include multiple adenomas, parathyroid hyperplasia, and parathyroid carcinoma. Biochemical screening of serum calcium detects PHPT early and reduce the classical presentation with renal stones or bone manifestations. Reported literature cases of PHPT described mainly of patients presenting with severe skeletal deformities, fractures and aesthesia, similar to this reported case $(8,9)$. In a large case series, the correct diagnosis was delayed for years because most of the patients presented with skeletal problems (30\%-40\%), such as osteopenia, osteoclastoma, fractures and renal involvement (40\%-50\%) e.g. nephrocalcinosis or renal calculi, both of which are the complications associated with long term hyperparathy- roidism (9). This case unusually presented with initial normocalcemia, much younger age and concomitant multiple fractures and brown tumors which raised diagnostic dilemma. Serra et al. reported that normocalcemic PHPT has similar complications like hypercalemic PHPT including nephrolithiasis and severe bone disease. Clinical presentation with pathological fractures as a symptom of hyperparathyroidism in young patient of parathyroid adenoma is extremely rare (10). Early diagnosis is important to prevent fracture.

Brown tumor is considered a benign tumor, belongs to giant-cell tumor group. Fibrous tissue replacing the bone is mixed with multinucleated giant cells and hemorrhagic foci (accumulation of hemosiderin in macrophages gives the name of the tumor). The lesion may grow, generate pain or pathological fractures. The majority of brown tumors are secondary to parathyroid adenoma, a small proportion secondary to parathyroid hyperplasia and rarely parathyroid carcinoma. Our patient presented with bone involvement, which has been noted in a number of other studies (3-4). 
Furthermore, plain radiographic images of the femur, pelvis, humerus and chest revealed multiple fractures and brown tumors caused by a high metabolism of bone and it's turnover, which has only infrequently been reported $(5,11)$.

The majority of parathyroid adenoma are hypersecretory and brown tumors have been described in such cases as a result of hyperparathyroidism. Fractures occur in almost $90 \%$ of untreated patients (5). If malignancy is suspected, the clinician should be highly alert to other possible causes of bony lesions. Brown tumors should be kept in mind during clinical practice. Multiple brown tumors may occur as a manifestation of primary hyperparathyroidism (12). In this case, decreased bone density and pathological fractures of left femur neck and shaft of right femur were obvious on the first visit. Nonspecific renal, neurologic, gastrointestinal, or bone and muscular symptoms may obscure the diagnosis. As was seen in the present case, a teen age girl was suffering more than 2 years without definite diagnosis.

The coexistence of a parathyroid tumor and thyroid disease is commonly misdiagnosed due to mutual interference during imaging in the early period of disease. The specificity and sensitivity of ultrasonography in the diagnosis of concurrent parathyroid tumor and thyroid nodules are low. The use of sestamibi scintigraphy for detecting parathyroid adenoma before an operation has improved the success of surgery because of it's accuracy. Despite its high sensitivity and specificity in the detection of parathyroid adenoma, SPECT alone is limited by its inability to provide anatomical details. Hybrid SPECT/ CT systems allow both functional and structural imaging performed in a single session with improved image quality compared to functional imaging alone. Kim et al. (13) evaluated the efficacy of the ${ }^{99 \mathrm{~m} T c-M I B I ~ S P E C T / C T}$ in minimally invasive parathyroidectomy comparing the abilities of ${ }^{99 \mathrm{~m} T c-M I B I}$ planar scintigraphy, SPECT and conventional imaging methods, including $\mathrm{CT}$ and ultrasonography to identify the precise locations of parathyroid adenomas and hyperplasias and found that 99mTc-MIBI SPECT CT was useful for minimally invasive

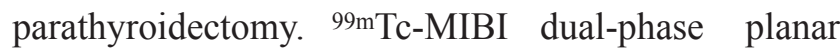
scintigraphy imaging produced an intrathyroidal image with a suspected intrahtyroidal adenoma in our patient. However, SPECT/CT found concentrated radioactivity inferior and posterior to the left thyroid lobe that was suspected to be an extrathyroid parathyroid adenoma; which was more clarified by the CT component. Additionally, CT component of the SPECT/CT study revealed multiple brown tumors that were negative on MIBI SPECT alone. Although MIBI scan showed false positive results (14), there are reports of negative MIBI scan in brown tumors.

Discrepant uptake among radiotracer of ${ }^{99 \mathrm{~m} T c-M I B I}$ and 99mTc-MDP in brown tumors has been reported in the literature (15). Changing manifestations of brown tumor examined with FDG, ${ }^{99 \mathrm{~m} T c-M I B I}$ and ${ }^{99 \mathrm{~m} T c-M D P}$ have been reported (16,17). A pattern of increased MDP uptake might be seen in bone scintigraphy representing reactive osteoblastic bones surrounding the lesions. Uptake of ${ }^{99 \mathrm{~m} T c-M I B I}$ has also been reported in brown tumors patients with parathyroid adenoma $(18,19)$. However, this patient did not display radiotracer uptake on 99mTc-MIBI scan brown tumor located in the right humeus, clavicles, ribs and femurs. Lack of mitochondria and differences in cellularity among brown tumors might be responsible for their non-accumulation of radiotracer. The negative MIBI scintigraphic findings in our patient are concordant with other researchers' results. Zanglis et al. reported no focal uptake of ${ }^{99 \mathrm{~m} T c-M I B I}$ in the brown tumors in a patient with spontaneous fracture (16). Multiple brown tumors without focal ${ }^{99 \mathrm{mTc}-M D P}$ or 99mTc-MIBI uptake were reported in a patient with secondary and tertiary hyperparathyroidism (20).

Interestingly, our patient had persistently elevated PTH level after parathyroidectomy although there was no evidence of recurrence or surgical failure on postoperative evaluation. This appears to be a response to severe hyperparathyroidism and vitamin D insufficiency. Vitamin D would decrease calcium absorption resulting in bone hunger and lead to excessive PTH secretion (21). Prior research has shown that patients with elevated PTH levels have lower preoperative 25-OHD levels $(22,23)$, as in our patient who had preoperative vitamin D insufficiency.

This interesting case emphasizes the importance of keeping primary hyperparathyroidism as a differential diagnosis when a young patient presents with a history of multiple pathological fractures history. Though rare, PHPT-causing brown tumors related skeletal fractures is an important differential diagnosis in the evaluation of patients presenting with multiple foci of radiotracer uptake on bone scan without an established primary neoplasm. 


\section{REFERENCES}

1. Kollars J, Zarroug AE, van Heerden J, Lteif A, Stavlo P, Suarez L, Moir C, Ishitani M, Rodeberg D. Primary hyperparathyroidism in pediatric patients. Pediatrics. 2005;115(4):974-980.

2. Deshmukh RG, Alsagoff SA, Krishnan S, Dhillon KS, Khir AS. Primary hyperparathyroidism presenting with pathological fracture. Journal of the Royal College of Surgeons of Edinburgh. 1998 Dec; 43(6):424-427.

3. Al-Sobhi S, Ashari LH, Ingemanssion S. Detection of metastatic parathyroid carcinoma with

Tc-99m sestamibi imaging. Clin Nucl Med. 1999; 24:21 - 23.

4. Chalmers J, Irvine GB. Fractures of the femoral neck in elderly patients with hyperparathyroidism. Clin Orthop Relat Res. 1988; 229:125-130.

5. Radulescu D, Chis B, Donca V, Munteanu V. Brown tumors of the femur and pelvis secondary to a parathyroid carcinoma: report of one case. Rev Med Chil. 2014; 142:919-923. doi: 10.4067/S0034-98872014000700014.

6. Wermers RA, Khosla S, Atkinson EJ, Achenbach SJ, Oberg AL, Grant CS, Melton LJ 3rd. Incidence of primary hyperparathyroidism in Rochester, Minnesota, 1993-2001: an update on the changing epidemiology of the disease. J bone Miner Res. 2006; 21:171-177.

7. Yeh MW, Ituarte PH, Zhou HC, et al. Incidence and prevalence of primary hyperparathyroidism in a racially mixed population. J Clin Endocrinol Metab. 2013; 98:1122-1129. doi: 10.1210/jc.2012-4022.

8. Rowlands BC. Hyperparathyroidism; an early historical survey. Ann R Coll Surg Eng. 1972; 51:81-90.

9. KrauszY, Bettman L, Guralnik L, Yosilevsky G, Keidar Z, Bar-ShaLom R, et al. Technetium-99m-MIBI SPECT/CT in primary hyperparathyroidism. World J Surg. 2006; 30:76-83.

10. Serra A, Bolasco P, Satta L, Nicolosi A, Uccheddu A, Piga M. Role of SPECT/CT in preoperative assessment of hyperparathyroid patients. Radiol Med. 2006; 111:999-1008.

11. Raccha R. Pathological fractures as a presenting symptom of parathyroid adenoma: a report of three cases. BJMP. 2008; 1:26-29.

12. Resendiz-Colosia JA, Alvarado-Cabrero I, Flores-Diciz R, et al. Multiple maxillofacial brown tumors as a primary hyperparathyroidism manifestation. Gaceta Medica de Mexico. 2008; 144: 155-160.

13. Kim YI, Jung YH, Hwang KT, Lee HY. Efficacy of 99mTc-Sestamibi SPECT/CT for minimally invasive parathyroidectomy: Comparative study with $99 \mathrm{mTc}$-sestamibi scintigraphy, SPECT, US and CT. Ann Nucl Med. 2012; 26:804-810.

14. Miyakoshi M, Kamoi K, Takano T, et al. Multiple brown tumors in primary hyperparathyroidism caused by an adenoma mimicking metastatic bone disease with false positive results on computed tomography and $99 \mathrm{mTc}$-sestamibi imaging: MR findings. Endocr J. 2007; 54:205-210.

15. Gahier Penhoat M, Drui D, Ansquer C, Mirallie E, Maugars Y, Guillot P. Contribution of 18-FDG PET/CT to brown tumor detection in a patient with primary hyperparathyroidism. Joint Bone Spine. 2017; 84:209-212. doi:10.1016/j.jbspin.2016.06.007.

16. Zanglis A, Andreaopoulos D, Zissimopoulos A et al. Multiple brown tumors with $99 \mathrm{mTc}$ MDP superscan appearance and negative 99mTc-MIBI uptake. Clin Nucl Med. 2006; 31:640-643.

17. Demir H, Halac M, Gorur GD, et al. FDG PET/CT findings in primary hyperparathyroidism mimicking multiple bone metastases. Eur J Nucl Med Mol Imag. 2008; 35:686.

18. Dinauer P, Balingit A, Rivera JE. Tc-99m-MIBI sestamibi imaging of brown tumors of primary hyperparathyroidism. Clin Nucl Med. 1996; 21:192-196.

19. Nahar N, Khan N, Mahmood S, et al. Multiple brown tumors in a normocalcimic patient with primary hyperparathyroidism - A case report. B J Nucl Med. 2019; 22:68-72.

20. Gedik GK, Ata O, Karabagli P, Sari O. Differential diagnosis between secondary and tertiary hyperparathyroidism in a case of a giant-cell and brown tumor containing mass. Findings by 99mTc -MDP, 18F-FDG PET/CT and 99mTc -MIBI scans. Hell J Nucl Med. 2014; 17:214-217. doi: 10.1967/s002449910147.

21. Walker MD, Cong E, Lee JA, Kepley A, Zhang C, McMahon DJ, Silverberg SJ. Vitamin D in primary hyperparathyroidism: Effects on clinical, biochemical and densitometric presentation. J Clin Endocrinol Metab. 2015; 100:3443-3451.

22. Oltman SC, Maalouf NM, Holt S. Significance of elevated parathyroid hormone after parathyroidectomy for primary hyperparathyroidism. Endocr Practice. 2011; 17:57-62.

doi: 10.4158/EP10324.RA.

23. Cao S, Hu Y, Zhao Y, et al. A retrospective study of elevated post-operative parathormone in primary hyperparathyroid patients. Oncotarget. 2017; 18:101158-101164. 